\title{
Squeezing of the Super-Elastic Double-Helix Photon in the Gravitational Field (Hidden in Very-Well Known Old Formulae). Pound - Rebka - Snider Effect Studied by the Advanced LIGO Instrument, Pioneer Anomaly and CMB (Cosmic Microwave Background) (23.11.2019)
}

\author{
Jiří Stávek ${ }^{1}$ \\ ${ }^{1}$ Bazovského, Prague, Czech Republic \\ Correspondence: Jiř́ Stávek, Bazovského 1228, 16300 Prague, Czech Republic. E-mail: stavek.jiri@seznam.cz \\ Received: November 22, 2019 \\ Accepted: November 30, 2019 \\ Online Published: December 2, 2019 \\ doi:10.5539/apr.v12n1p8 \\ URL: http://dx.doi.org/10.5539/apr.v12n1p8
}

\begin{abstract}
In our approach we have combined knowledge of Old Masters (working in this field before the year 1905), New Masters (working in this field after the year 1905) and Dissidents under the guidance of Louis de Broglie and David Bohm. Based on the great experimental work of Robert Pound, Glen A. Rebka and J.L. Snider we have proposed a squeezing of the super-elastic double-helix photon in the gravitational field. We have newly defined the squeeze rate of that photon particle on the helical path. We have inserted this squeeze rate into the very-well old formulae of Newton, Soldner, Gerber and Einstein and might glimpse traces of the quantum gravity. The squeeze rate of photons can be studied in details using the Great instrument - the Advanced LIGO - located on the surface of the Earth (USA, Italy, Japan). The observed strains on the level $5^{*} 10^{-19}$ should be caused by the gravitational field of our Earth. The observed strains on the level $5^{*} 10^{-22}$ should be caused by the gravitational fields of the Moon and the Sun. We estimate that the experimental value of the gravitational constant G studied by the LIGO instrument can achieve the accuracy to the level of ppb (parts per billion) after the removal of those strains from the measured signal and the removal of the gravitational influences of the Earth, the Moon, the Sun, Venus and Jupiter. To study the squeeze effect on a bigger scale we propose to analyze the Pioneer anomaly where Pioneer's photons have been flying around the planets in our Solar system causing the squeeze effect - the anomalous blueshift. Similarly, we can study cosmic microwave photons flying around the objects in our Solar system that might create "the axis of evil" - temperature fluctuations in the CMB map (Wien displacement law). Can we prepare in our Solar system "tired" light by frequent blueshift - redshift transitions? Can it be that Nature cleverly inserted the squeeze rate into our very-well known Old Formulae? We want to pass this concept into the hands of Readers of this Journal better educated in the Mathematics and Physics.
\end{abstract}

Keywords: Super-Elastic Double-Helix Photon, Squeeze Effect in the Gravitational Field, Squeeze Rate Hidden in Old Formulae, Pound-Rebka-Snider Experiment, Advanced Ligo, Big G and Advanced Ligo, Pioneer Anomaly, Cosmic Microwave Background, "The Axis of Evil", "Tired" Light

\section{Introduction}

The famous quote of Heraclitus "Nature loves to hide" was described in details by Pierre Hadot in 2008. Hadot in his valuable book gives us many examples how Nature protects Her Secrets. In several situations the enormous research of many generations is strongly needed before the right "recipe" unlocking the True reality can be found. Johann Wolfgang Goethe remarked to our research: "Nature does not suffer Her veil to be taken from Her, and what She does not choose to reveal to the spirit, thou wilt wrest from Her by levers and screws." In our model of the photon we have found that Nature could keep Her Secrets hidden in plain sight and thus can perfectly document Her Top Art of Hiding.

Cylindrical Helix represents one of the most fascinating curves in Nature and Science and is among the oldest curves. Cylindrical Helix belongs to the Treasure of Geometry. The Cylindrical Helix seem to have been discovered and thoroughly studied by Apollonius of Perga (The Great Geometer) and his scholars as e.g. Geminus of Rhodes. Cylindrical helix is composed from two motions - circular and translational. Heron 
discovered the cylindrical helix construction by triangle wrapping that gives to us a deeper view in the properties of the elastic helical WAVE. For the details of the Heron's construction see P. Mancosu and A. Arana (2010).

The model of the double helix for the description of the photon wave appeared many times in the so-called dissident literature (outside of the mainstream literature). There are known many proposals for this double helix composition. Louis de Broglie proposed at the 1927 Solvay Conference his model of the full wave and the pilot guiding wave but could not give a deeper physical interpretation of his concept. Later Louis de Broglie (1939) proposed two component model of the photon. (Many modern Dissidents continue to develop this double-helix model of the photon where both helical paths are occupied by particles). In 1952 David Bohm rediscovered this pilot wave model and developed it as the de Broglie - Bohm theory. Since that time the concept of empty guiding waves remains still open and has been waiting for the physical interpretation. See J.S. Bell in 1992, L. Hardy in 1992, P.J. Lewis in 2007, W. Seager in 2018, and many others.

What is the behavior of the super-elastic double-helix photon in the gravitational field? Can we describe the elasticity of this double-helix in the gravitational field?

In order to achieve our target, we have combined knowledge of Old Masters (working in this field before the year 1905), New Masters (working in this field after the year 1905), and Dissidents working on the double helix model of the photon for many years. The key breakthrough was found in the works of Robert Pound, Glen A. Rebka and J.L. Snider who used the expression $v=g h / c$ where $v$ is an un-known effective speed, $g$ is the acceleration, $\mathrm{h}$ is the distance, and $\mathrm{c}$ is the light speed.

(We are aware of the famous quote of Richard Feynman: "Gravity discussions: it is not good for my blood pressure.")

\section{Inspirations from Old Masters and New Masters - The Squeeze Rate of Photons in the Gravitational Field}

We were inspired by many Great Researchers working in this field for generations. The key breakthrough was found in the works of Robert Pound, Glen A. Rebka and J.L. Snider who used the expression v $=\mathrm{gh} / \mathrm{c}$ where $v$ is an un-known effective speed, $\mathrm{g}$ is the acceleration, $\mathrm{h}$ is the distance, and $\mathrm{c}$ is the light speed.

Table 1. Inspirations from Great Masters to formulate the squeeze rate of photons in the gravitational field

POUND - REBKA - SNIDER's squeeze rate for the small height difference

$v_{\text {squeеzе }}=\frac{g h}{c}$

Squeeze rate for the distance $\mathrm{R}$ from the source mass $\mathrm{M}$

$V_{\text {squeeze }}=\frac{2 G M}{R c}$

Squeeze rate on the circular path at the distance $\mathrm{R}$ from the source mass $\mathrm{M}$

$U_{\text {squeeze }}=\frac{G M}{R c}$

Squeeze rate on the planet orbit

$W_{\text {squeeze }}=\frac{3 G M}{R c}$

SCHWARZSCHILD radius $\mathrm{R}_{\mathrm{S}}$ is the distance from the source mass $\mathrm{M}$ where $\mathrm{V}_{\text {squeeze }}=\mathrm{c}$

$R_{S}=\frac{2 G M}{c} \frac{1}{c}$

NEWTON's gravitation law

$F=G \frac{M m}{R^{2}}=\frac{G M}{R c} \frac{m c}{R}=U_{\text {squeeze }} \frac{m c}{R}$

Frank WILCZEK: „, It would be a bracing achievement, and major progress, to identify any concrete, observable phenomenon that brings in truly characteristic features of quantum gravity beyond the semiclassical approximation in common use. Actual observation would bring the subject to another level". 
We will try to apply this model of the squeeze rate of photons in the gravitational field into the already very-well situations and will insert this concept into the Old Formulae. On the other hand, there are several situations where we can collect experimental data with the existing most advanced technology - advanced LIGO, Pioneers missions, CMB missions to support or to reject this scenario.

\section{Redshifted Pound - Rebka - Snider Experiments}

In these experiments the super-elastic double-helix photon emanates from the field mass $\mathrm{M}$ to the distance $\mathrm{R}$ from the center of the gravitational field. In the Pound - Rebka - Snider experiment the photon travels through a short distance $\mathrm{h}$ from the position 1 to the position 2 .

The longitudinal photon wavelength $\lambda_{\mathrm{R}}$ is redshifted as:

$$
\frac{\lambda_{R}}{\lambda_{0}}=\frac{1}{\sqrt{1-\frac{2 G M}{R c} \frac{1}{c}}} \approx 1+\frac{G M}{R c} \frac{1}{c}+\frac{3}{8}\left(\frac{2 G M}{R c} \frac{1}{c}\right)^{2}+\ldots
$$

The photon frequency $v_{\mathrm{R}}$ is decreased as:

$$
\frac{\nu_{R}}{\nu_{0}}=\sqrt{1-\frac{2 G M}{R c} \frac{1}{c}} \approx 1-\frac{G M}{R c} \frac{1}{c}-\frac{1}{8}\left(\frac{2 G M}{R c} \frac{1}{c}\right)^{2}+\ldots
$$

The period $T_{R}$ measured by that redshifted photon wave at the distance $\mathrm{R}$ from the source of gravitation equals:

$$
\frac{T_{R}}{T_{0}}=\frac{1}{\sqrt{1-\frac{2 G M}{R c} \frac{1}{c}}} \approx 1+\frac{G M}{R c} \frac{1}{c}+\frac{3}{8}\left(\frac{2 G M}{R c} \frac{1}{c}\right)^{2}+\ldots
$$

The amplitude $\mathrm{A}_{\infty}$ of that redshifted photon wave shrinks as:

$$
\frac{A_{R}}{A_{0}}=\frac{\lambda_{R}}{2 \pi} \frac{2 \pi}{\lambda_{0}}=\sqrt{1-\frac{2 G M}{R c} \frac{1}{c}} \approx 1-\frac{G M}{R c} \frac{1}{c}-\frac{1}{8}\left(\frac{2 G M}{R c} \frac{1}{c}\right)^{2}+\ldots
$$

Robert Pound, Glen A. Rebka and J.L. Snider collected data for a small height $\mathrm{h}$ where an analogy with the relativistic Doppler formula can be used:

$$
\frac{\lambda_{2}}{\lambda_{1}}=\frac{\sqrt{1+\frac{g h}{c} \frac{1}{c}}}{\sqrt{1-\frac{g h}{c} \frac{1}{c}}} \approx 1+\frac{g h}{c} \frac{1}{c}+\frac{1}{2}\left(\frac{g h}{c} \frac{1}{c}\right)^{2}+\ldots
$$

and:

$$
\frac{\nu_{2}}{\nu_{1}}=\frac{\sqrt{1-\frac{g h}{c} \frac{1}{c}}}{\sqrt{1+\frac{g h}{c} \frac{1}{c}}} \approx 1-\frac{g h}{c} \frac{1}{c}+\frac{1}{2}\left(\frac{g h}{c} \frac{1}{c}\right)^{2}+\ldots
$$

\section{Blueshifted Pound - Rebka - Snider Experiments}

In these experiments the super-elastic double-helix photon emanates from the "infinity" to the distance R toward the field mass M. In the Pound - Rebka - Snider experiment the photon travels through a short distance $\mathrm{h}$ from the position 2 to the position 1 .

The longitudinal photon wavelength $\lambda_{R}$ at the distance $\mathrm{R}$ is blueshifted as: 


$$
\frac{\lambda_{R}}{\lambda_{\infty}}=\sqrt{1-\frac{2 G M}{R c} \frac{1}{c}} \approx 1-\frac{G M}{R c} \frac{1}{c}-\frac{1}{8}\left(\frac{2 G M}{R c} \frac{1}{c}\right)^{2}+\ldots
$$

The photon frequency $v_{\mathrm{R}}$ at the distance $\mathrm{R}$ is increased as:

$$
\frac{\nu_{R}}{\nu_{\infty}}=\frac{1}{\sqrt{1-\frac{2 G M}{R c} \frac{1}{c}}} \approx 1+\frac{G M}{R c} \frac{1}{c}+\frac{3}{8}\left(\frac{2 G M}{R c} \frac{1}{c}\right)^{2}+\ldots
$$

The period $T_{R}$ measured by that blueshifted photon wave in the distance $R$ equals:

$$
\frac{T_{R}}{T_{\infty}}=\sqrt{1-\frac{2 G M}{R c} \frac{1}{c}} \approx 1-\frac{G M}{R c} \frac{1}{c}-\frac{1}{8}\left(\frac{2 G M}{R c} \frac{1}{c}\right)^{2}+\ldots
$$

The amplitude $A_{R}$ of that blueshifted photon wave expands as:

$$
\frac{A_{R}}{A_{\infty}}=\frac{\lambda_{R}}{2 \pi} \frac{2 \pi}{\lambda_{\infty}}=\frac{1}{\sqrt{1-\frac{2 G M}{R c} \frac{1}{c}}} \approx 1+\frac{G M}{R c} \frac{1}{c}+\frac{3}{8}\left(\frac{2 G M}{R c} \frac{1}{c}\right)^{2}+\ldots
$$

Robert Pound, Glen A. Rebka and J.L. Snider collected data for a small height $\mathrm{h}$ where an analogy with the relativistic Doppler formula can be used. The photons fly from the position 2 towards the position 1:

$$
\frac{\nu_{1}}{\nu_{2}}=\frac{\sqrt{1+\frac{g h}{c} \frac{1}{c}}}{\sqrt{1-\frac{g h}{c} \frac{1}{c}}} \approx 1+\frac{g h}{c} \frac{1}{c}+\frac{1}{2}\left(\frac{g h}{c} \frac{1}{c}\right)^{2}+\ldots
$$

and:

$$
\frac{\lambda_{1}}{\lambda_{2}}=\frac{\sqrt{1-\frac{g h}{c} \frac{1}{c}}}{\sqrt{1+\frac{g h}{c} \frac{1}{c}}} \approx 1-\frac{g h}{c} \frac{1}{c}+\frac{1}{2}\left(\frac{g h}{c} \frac{1}{c}\right)^{2}+\ldots
$$

\section{Blueshifted Photons on a Circular Path around the Field Mass M - Advanced LIGO Instrument on the Surface of the Earth}

In these experiments the super-elastic double-helix photons have been created on a circular path around the source of gravity with the mass M. In this case the gravitational field modifies the amplitude of those photons. It is a very minute effect but thanks to the Great Advanced LIGO instrument we can extract valuable experimental data to estimate this model.

The amplitude $A_{R}$ of these photons expands as:

$$
\frac{A_{R}}{A_{0}}=\frac{\lambda_{R}}{2 \pi} \frac{2 \pi}{\lambda_{0}} \frac{1}{\sqrt{1-\frac{G M}{R c} \frac{1}{c}}} \approx 1+\frac{1}{2} \frac{G M}{R c} \frac{1}{c}+\frac{3}{8}\left(\frac{G M}{R c} \frac{1}{c}\right)^{2}+\ldots
$$

The photon wave on a circular path is longitudinally blueshifted as $\lambda_{R}$ :

$$
\frac{\lambda_{R}}{\lambda_{0}}=\sqrt{1-\frac{G M}{R c} \frac{1}{c}} \approx 1-\frac{1}{2} \frac{G M}{R c} \frac{1}{c}-\frac{1}{8}\left(\frac{G M}{R c} \frac{1}{c}\right)^{2}+\ldots
$$


The photon frequency $v_{R}$ on a circular path is increased as:

$$
\frac{\nu_{R}}{\nu_{0}}=\frac{1}{\sqrt{1-\frac{G M}{R c} \frac{1}{c}}} \approx 1+\frac{1}{2} \frac{G M}{R c} \frac{1}{c}+\frac{3}{8}\left(\frac{G M}{R c} \frac{1}{c}\right)^{2}+\ldots
$$

The period $\mathrm{T}_{\mathrm{R}}$ of that photon on a circular path equal:

$$
\frac{T_{R}}{T_{0}}=\sqrt{1-\frac{G M}{R c} \frac{1}{c}} \approx 1-\frac{1}{2} \frac{G M}{R c} \frac{1}{c}-\frac{1}{8}\left(\frac{G M}{R c} \frac{1}{c}\right)^{2}+\ldots
$$

We can make the first test of this concept - we will assume that the gravitational field of our Earth will modify the amplitude of laser photons on the helical path with the amplitude $A_{0}=\lambda_{0} / 2 \pi$. The photons used in the advanced LIGO instrument have the wavelength $\lambda_{0}=1064 \mathrm{~nm}$. For the of wavelength difference of the original photon $\lambda_{0}$ and the blueshifted photon $\lambda_{\mathrm{R}}$ we should observe some oscillations in the range of $2.327^{*} 10^{-15} \mathrm{~m}$ :

$$
\lambda_{0}-\lambda_{R}=2 \pi \lambda_{0}\left(\frac{A_{R}}{A_{0}}-1\right) \approx 2 \pi \lambda_{0} \frac{1}{2} \frac{G M}{R c} \frac{1}{c} \approx 2.327 * 10^{-15} \quad \mathrm{~m}
$$

There are many specialists analyzing the signals taken from the advanced LIGO experiments. See the web sites of the LIGO Project, VIRGO Project and KAGRA Project.

There are two visible strains $\mathrm{h}_{1}=5^{*} 10^{-19}$ and $\mathrm{h}_{2}=5^{*} 10^{-22}$ in the LIGO data. If we multiply those strains by 4000 $\mathrm{m}$ (the length of the used Fabry - Pérot cavity) we will get oscillations with the size $\sim 2 * 10^{-15} \mathrm{~m}$ and $\sim 2 * 10^{-18} \mathrm{~m}$.

It is interesting coincidence of the strain $\mathrm{h}_{1}=5^{*} 10^{-19}$ (length difference oscillations $\sim 2 * 10^{-15} \mathrm{~m}$ ) with the gravitational action of our Earth on the photons used in the Fabry - Pérot cavity in the advanced LIGO instrument.

The second strain $\mathrm{h}_{2}=5^{*} 10^{-22}$ (length difference oscillations $\sim 2 * 10^{-18} \mathrm{~m}$ ) might be caused by the gravitation actions of the Moon and the Sun. We want to pass this model into the hands of experienced Readers of this Journal.

It will be interesting to compare strains obtained in the near future by KAGRA (located in the tunnel 200 meters below the Mt. Ikenoyama with a slope $0.3 \%$ ) with strains obtained by LIGO and VIRGO located on the surface. There might be difference in the local gravitation fields and in the slopes of those long tubes (Pound - Rebka Snider effect).

6. Advanced LIGO Instrument on the Surface of the Earth and KAGRA Located 200 Meters below the Surface: The Determination of the Gravitational Constant G

There were done enormous experimental works in the field of the determination of the of the gravitational constant G in the last decade - for reviews see "The Theo Murphy Meeting" (2014), C. Rothleitner and S. Schlamminger (2017), J. Wu et al. (2019).

There are two Groups working in this field with the Fabry - Pérot cavity - the Wuppertal school (e.g., the Great $\mathrm{PhD}$ Thesis of Achim Schumacher (1999) and Ulf Kleinevo $\beta$ (2002)) and the Great work of the Colorado school (Harold V. Parks and James E. Faller (2010)).

Akio Kawasaki (2019) proposed to use the Advanced LIGO instrument for the determination of the gravitational constant big G.

It could be interesting if these Great Experimental Masters working with the Advanced LIGO, VIRGO and KAGRA could share know-how and instruments with Teams measuring the Big G value. In this co-operation we might improve the big $\mathrm{G}$ accuracy to the range of ppb (parts per billion).

\section{Pioneer Anomaly - Blueshifted Pioneer Photons Modified by the Gravitational Fields of Planets in the Solar System}

John D. Anderson and his Team analyzed the Pioneer anomaly - blueshifted photons coming from Pioneers 10 and 11 that could not be explained with the known physics. There is a huge literature on this subject. Pioneers 10 and 11 during their path came close to several planets in the Solar system. 
We propose to analyze the effect of gravitational fields of planets in the Solar system on the amplitudes of those blueshifted photons based on the experience of the Advanced LIGO instrument.

\section{CMB Map - the Axis of Evil - Blueshifted CMB Photons Modified by the Gravitational Fields of Objects in the Solar System}

Cosmic microwave background is very well-known phenomenon with some exceptions. E.g., Erich Regener's prediction (in 1933) of the microwave background with the $\mathrm{T}=2.8 \mathrm{~K}$ is not known. E.g., A.K.T. Assis and M.C.D. Neves (1995).

Based on the data of Teams of COBE, WMAP and Planck Surveyor we know very well the temperature fluctuations of the CMB. One interesting anomaly is the so-called the "axis of evil" as it was coined by Kate Land and João Magueijo (2005).

We propose to analyze the effect of gravitational fields of objects in the Solar system on the amplitudes of those blueshifted photons based on the experience of the Advanced LIGO instrument. The time and position of objects in the Solar system might draw that "axis of evil" in the CMB map. Cosmic background photons on their long journey through the Space might be frequently slightly redshifted and blueshifted (see the next Chapter on the Soldner and Einstein Old Formula).

\section{Decomposition of the Old Formula of Soldner and Einstein - the Bending of Light by the Gravitational Field}

Johann Georg von Soldner in 1801 derived one half of the Formula and Albert Einstein in 1916 published the right complete Formula describing the deflection of the light:

$$
\theta=\frac{4 G M}{R c^{2}}
$$

In our approach we will decompose this Formula as follows:

$$
\theta=\frac{2 G M}{R c} \frac{1}{c}+\frac{2 G M}{R c} \frac{1}{c}=\frac{V_{\text {squeeze }}}{c}+\frac{V_{\text {squeeze }}}{c}=\tan \frac{\theta}{2}+\tan \frac{\theta}{2} \approx \frac{\theta}{2}+\frac{\theta}{2}=\theta
$$

The interpretation in words: the blueshifted photons approaching the vertex of the hyperbola - its amplitude expands with the squeeze rate $\mathrm{V}_{\text {squeeze }}$, behind the vertex the amplitude shrinks with the squeeze rate $\mathrm{V}_{\text {squeeze. }}$. In a far distance, the photon recovers its original wavelength $\lambda_{0}$ and amplitude $A_{0}$ by the redshift. If the photon is disturbed by another gravitational field, then this process - blueshift + redshift - is repeated. At this moment we do not know time needed for the full recovery - it can be studied by the Pioneer photons and/or photons from the microwave background. We do not know how many times this process can be repeated before the photon becomes "tired".

\section{0. "Tired" Light after Many Blueshift - Redshift Cycles}

It could be interesting to study repeatedly blueshift - redshift transitions of the super-elastic double-helix photons in our Solar system. Until now there are no experimental data to evaluate this effect. Can it happen that after many blueshift - redshift cycles the observed photon will be "tired"? (E.g., Fritz Zwicky in 1929).

At this moment we have available the Advanced LIGO instrument with the Fabry - Pérot cavity where we can bounce laser beam back and forth along the full $4 \mathrm{~km}$ length of each arm about 400 times before it is merge with other beam. We propose to compare strains obtained for "fresh" beams bounced only once with strains obtained from the "tired" photons.

\section{Decomposition of the Old Formula of Paul Gerber - the Anomalous Advance of the Perihelion of Mercury}

Paul Gerber in 1898 derived the correct expression of the Formula for the anomalous advance of the perihelion of Mercury. Albert Einstein in 1916 published the same Formula describing this event in the Elastic Universe:

$$
\theta=\frac{6 \pi G M}{R c^{2}} \quad \text { where } \quad R=a\left(1-\varepsilon^{2}\right) \quad \text { is the semilatus rectum }
$$

In our model we assume that redshifted gravitons push the planet while they are longitudinally oriented to the 
atoms of that planet. On the other side they brake the planet while they are attached to the atoms by their amplitudes. We can express this situation in a model by the Equation 21:

$$
\begin{gathered}
P=\frac{m c \lambda_{R}}{m c \lambda_{0}}-\frac{m c A_{R}}{m c A_{0}}=\frac{m c \lambda_{0}}{m c \lambda_{0}} \frac{1}{\sqrt{1-\frac{3 G M}{R c} \frac{1}{c}}}-\frac{m c \frac{\lambda_{0}}{2 \pi}}{m c \frac{\lambda_{0}}{2 \pi}} \sqrt{1-\frac{3 G M}{R c} \frac{1}{c}}=\frac{\frac{3 G M}{R c} \frac{1}{c}}{\sqrt{1-\frac{3 G M}{R c} \frac{1}{c}}} \approx \frac{3 G M}{R c} \frac{1}{c} \\
\theta \approx 2 \pi \frac{3 G M}{R c} \frac{1}{c}=2 \pi \frac{W_{\text {squeeze }}}{c}=2 \pi \tan \alpha \approx 2 \pi \alpha
\end{gathered}
$$

The anomalous advance of the perihelion of Mercury is caused by the co-operation of super-elastic gravitons that are trigonometrically organized in the volume of the planet. The squeezing effect of quantum particles might be behind the mechanism of this experimental observation. The action of this squeezing mechanism influences the planet motion on the whole ellipse $(2 \pi)$.

\section{Conclusions}

1. We have combined knowledge of Old Masters, New Masters, and Dissidents in order to newly formulate events in the gravitational fields.

2. We have defined the squeeze rate of the super-elastic double-helix photons.

3. We have inserted the squeeze rate formula into the Old Formulae of Newton, Soldner, Gerber and Einstein.

4. We have formulated properties of photons in Pound - Rebka - Snider experiments and in the gravitational field.

5. The strains in the LIGO data were interpreted as the influence of the Earth, Moon and Sun on the amplitude of laser photons.

6. Advanced LIGO might significantly improve the accuracy in the determination of the gravitational constant.

7. Pioneer anomaly can be explained by the action of gravitational fields of planets in our Solar system on the amplitude of Pioneer photons.

8. The "axis of evil" observed in the CMB map can be explained by the action of gravitational fields of planets in our Solar system on the amplitude of those cosmic photons.

9. We propose to study repeated blueshift - redshift cycles with photons in our Solar system to check the "tiredness" of photons. Advanced LIGO instrument can create "tired" photons by their numerous bouncing in the Fabry - Pérot cavity.

10. Nature might hide Her Beauty in plain sight protected by the mathematical camouflage.

11. Are there some more "hidden curves" in the Plato's Realm connected to the Photon and Electron Secrets? How to distinguish the real physical meaning written in those curves from fictious events if both are mathematically correct? How to work with the mathematical camouflage used by Nature to protect Her Secrets?

12. We want to pass this model into hands of Readers of this Journal better educated in Mathematics and Physics.

\section{Acknowledgments}

This work was supported by the JP\&FŠ Agency (Contract Number 25g/1963), by the VZ\&MŠ Agency (Contract Number 16000/1989) and by the GMS Agency (Contract Number 69110/1992). We were supported by the contract numbers $28101918 / 2018$ and 58287/2019. We have found the valuable support on the web site www.wolframalpha.com with the corrections of used formulae.

\section{Conflict of interests}

The authors declare that there is no conflict of interests regarding the publication of this paper.

\section{References}

Anderson et al. (2005). Study of the anomalous acceleration of Pioneer 10 and 11. Arxiv: gr-qc/0104064.v5. Apollonius of Perga. On the Cochlias. In L. Euclid (Ed.), Proclus. Friedlein, 105, 1-6.

Ashworth, R. A. (1998). Confirmation of Helical Travel of Light through Microwave Waveguide Analysis. Physics Essays, 11, 1-10. Retrieved from https://www.researchgate.net/publication/260829054 Confirmation_of_Helical_Travel_of_Light_through_Microwave_Waveguide_Analyses 
Assis, A. K. T., \& Neves, M. C. D. (1995). The Redshift Revisited. Astrophysics and Space Science, 227, 13-24. Retrieved from https://www.ifi.unicamp.br/ assis/Astrophys-Space-Sci-V227-p13-24(1995).pdf

Axis of Evil (structure in the CMB map). Retrieved from https://en.wikipedia.org/wiki/Axis_of_evil_(cosmology)

Bardini, G., \& Gianella, G. M. (2016). A Historical Walk along the Idea of Curvature, from Newton to Gauss Passing from Euler. International Mathematical Forum, 11, 259-278. Retrieved from http://www.m-hikari.com/imf/imf-2016/5-8-2016/p/bardiniIMF5-8-2016.pdf

Bell, J. S. (1992). Six Possible Worlds of Quantum Mechanics. Foundations of Physics, 22, 1201-1215.

Blaschke, P. (2017). Pedal Coordinates, Dark Kepler and Other Forces Problems. Arxiv: 1704.00897v1.

Blåsjö, V. (2017). Transcendental Curves in the Leibnizian Calculus. Academic Press, Elsevier, ISBN: 978-0-12-813237-1.

Blåsjö, V. (2018). Mathematicians Versus Philosophers in Recent Work on Mathematical Beauty. Journal of Humanistic Mathematics, 8, 414-431. Retrieved from https://scholarship.claremont.edu/cgi/viewcontent. cgi?referer=https://www.google.com/\&httpsredir=1\&article=1380\&context=jhm

Bohm, D. (1952). A Suggested Interpretation of the Quantum Theory in Terms of Hidden Variables, I. Physical Review, 85, 166-179.

Bohm, D. (1989). Quantum Theory. Dover Publications, ISBN-10: 9780486659695.

Böhm, J. (2016). Wonderful World of Pedal Curves. Retrieved from http://rfdz.ph-noe.ac.at/fileadmin/Mathematik_Uploads/ACDCA/TIME2016/Boehm_Pedals_.pdf

Brackenridge, J. B. (1996). The Key to Newton's Dynamics: The Kepler Problem and the Principia. Berkeley: University of California Press. ISBN: 978-0520202177.

Bruneau, O. (2015). ICT and History of Mathematics: The Case of the Pedal Curves from 17th Century to 19th Century. Retrieved from https://hal.archives-ouvertes.fr/hal-01179909/document

Cohen et al. (2019). Realism and Causality I: Pilot Wave and Retrocausal Models as Possible Facilitators. Arxiv: $1902.05108 \mathrm{v} 2$.

Cohen, I. B. (1999). A Guide to Newton's Principia. The Principia: The Mathematical Principles of Natural Philosophy. Berkeley, CA, University California Press. ISBN: 978-0-520-08816-0.

Cohen, I. B., \& Smith, G. E. (Eds.) (2004). The Cambridge Companion to NEWTON. Cambridge University Press, Cambridge. ISBN 0-521-65696-6.

Cook, T. A. (1914). The Curves of Life. Constable and Company, London.

Cosmic Microwave Background. Retrieved from https://en.wikipedia.org/wiki/Cosmic_microwave_background

Cylindrical Helix. Retrieved from http://demonstrations.wolfram.com/SineAndCosineHelix/

Cylindrical Helix. Retrieved from http://mathworld.wolfram.com/Helix.html

Cylindrical Helix. Retrieved from https://en.wikipedia.org/wiki/Helix

Cylindrical Helix. Retrieved from https://www.mathcurve.com/courbes3d.gb/helicecirculaire/ helicecirculaire.shtml

Darrigol, O. (2012). A History of Optics. From Greek Antiquity to the Nineteenth Century. Oxford University Press. Oxford. ISBN-10: 0199644373.

De Broglie, L. (1927). Electrons et Photons: Rapport et Discussions du Cinquième Conseil de Physique tenu à Bruxelles du 24 au 29 Octobre 1927. Gauthier-Villars.

De Broglie, L. (1939). Matter and light: The New Physics (pp. 136-142). W.W: Norton CO., New York.

De Broglie, L. (n. d.). Bohm Theory. Retrieved from https://en.wikipedia.org/wiki/De_Broglie\% E2\%80\%93Bohm_theory

Dennis, D. (1995). Historical Perspectives for the Reform of Mathematics Curriculum: Geometric Curve Drawing Devices and their Role in the Transition to an Algebraic Description of Functions. Dissertation at Cornell University. Retrieved from http://www.quadrivium.info/mathhistory/CurveDrawingDevices.pdf

Dürer, A. (1525). Underweysung der Messung, mit dem Zirckel und Richtscheyt, in Linien, Ebenen und gantzen corporen. Dürer's Plan for the Cylindrical Helix in a Staircase. Retrieved from https://commons.wikimedia.org/wiki/Category:Underweysung_der_Messung 
Dürr, D., \& Teufel, S. (2009). Bohmian Mechanics: The Physics and Mathematics of Quantum Theory (Fundamental Theories of Physics). Springer, ISBN 10-3540893431.

Einstein, A. (1916). The Foundation of the General Theory of Relativity. Annalen der Physik, 49, 769-822. Retrieved from https://en.wikisource.org/wiki/The_Foundation_of_the_Generalised_Theory_of_Relativity

Einstein, A. (1920). Relativity: The Special and the General Theory. Reprint (2015) Martino Fine Books, ISBN-10: 1891396304.

Evans, R. (2014). Cosmic Microwave Background: How it Changed Our Understanding of the Universe. Springer, ISBN-10:3319099272.

Feynman, R. (1964). Quantum Behavior. Feynman Lectures, Vol III., Chapter 1. Retrieved from http://www.feynmanlectures.caltech.edu/III_01.html

Freire, O. (2003). Science and Exile: David Bohm, the Hot Times of the Cold War, and his Struggle for a New Interpretation of Quantum Mechanics. Retrieved from https://arxiv.org/ftp/physics/papers/0508/0508184.pdf

Freire, O. (2015). The Quantum Dissidents: Rebuilding the Foundations of Quantum Mechanics (1950-1990). With a Forword by S.S. Schweber. Springer, ISBN-10: 9783662446614.

Fried, M., \& Unguru, S. (2001). Apollonius of Perga's Conica: Text, Context, Subtext. Mnemosyne, Bibliotheca Classic. ISBN-10: 9004119779.

Gant, de F. (1995). Force and Geometry in Newton's Principia. Princeton University Press, Princeton. ISBN 0-691-03367-6.

Gauthier, R. (2017). Entangled Double-Helix Superluminal Composite Photon Model Defined by Fine Structure Constant. Retrieved from https://www.researchgate.net/publication/320840733_Entangled_Double-Helix_S uperluminal_Composite_Photon_Model_Defined_by_Fine_Structure_Constant

Geminus of Rhodes: Cylindrical Helix. Retrieved from https://www-history.mcs.st-and.ac.uk/Biographies/ Geminus.html

Gerber, P. (1898). Die räumliche und zeitliche Ausbreitung der Gravitation. Zeitschrift für Mathematik und Physik, 43, 93-104. Retrieved from http://www.mahag.com/fremd/gerber.htm

Giné, J. (2005). On the Origin of the Anomalous Precession of Mercury's Perihelion. Arxiv: physics/0510086v1.

Giné, J. (2006). On the Origin of the Deflection of Light. Arxiv: physics/0512121v3.

Guicciardini, N. (2003). Reading the Principia: The Debate on Newton's Mathematical Methods for Natural Philosophy from 1687 to 1736. Cambridge University Press, Cambridge. ISBN-10: 0521544033.

Hadot, P. (2008). The Veil of Isis: An Essay on the History of the Idea of Nature. Belknap Press. ISBN-10: 0674030494.

Hall, A. R. (2015). Philosophers at War: The Quarrel Between Newton and Leibniz. Cambridge University Press. ISBN: 052152489X.

Hardy, L. (1992). On the Existence of Empty Waves in Quantum Theory. Physics Letters A, 167, 11-16.

Hawking, S. W., \& Israel, W. (1987). Three Hundred Years of Gravitation. Cambridge University Press, ISBN-10:0521343127.

Heat, T. L. (2015). Apollonius of Perga: Treatise on Conic Sections. Carruthers Press. ISBN-10: 1446021262.

Helical Electromagnetic Waves. (2011). Retrieved from http://blackholeformulas.com/files/EnM.html

Heron. (1912). Heronis Alexandrini opera quae supersunt omnia. Teubner, Leipzig, Ed. And translated into German by J.L. Heiberg.

Huygens, Chr. (1678). Traité de la Lumière, Leyden, Van der Aa. (In Treatise on Light, London: McMillan, 1912).

Interpretations of Quantum Mechanics. Retrieved from https://en.wikipedia.org/wiki/Interpretations_of_ quantum_mechanics

Jaki, S. L. (1978). Johann Georg von Soldner and the Gravitational Bending of Light. Foundations of Physics, 8, 927-950.

KAGRA. Retrieved from https://gwcenter.icrr.u-tokyo.ac.jp/en/ 
Kawasaki, A. (2019). Measurement of the Newtonian Constant of Gravitation by Precision Displacement Sensors. Arxiv: $1903.11223 \mathrm{v} 1$.

Kleinevo $\beta$, U. (2002). Bestimmung der Newtonschen Gravitationskonstanten G. PhD Thesis, University Wuppertal, WUB-DIS-2002-2.

Köller, J. (2016). Fusspunktkurven und Gegenfusspunktkurven. Retrieved from http://www.mathematischebasteleien.de/fusspunktkurve.htm

Kvasz, L. (2012). Galileo, Descartes, and Newton - Founders of the Language of Physics. Acta Physica Slovaca, 62, 519-614. Retrieved from http://www.physics.sk/aps/pubs/2012/aps-12-06/aps-12-06.pdf

Laird, W. R., \& Roux, S. (Eds.) (2008). Mechanics and Natural Philosophy before the Scientific Revolution. Springer. ISBN 978-90-481-7491-1.

Land, K., \& Magueijo, J. (2005). The Axias of Evil. Arxiv: 0502237v2.

Le Verrier, U. (1859). Lettre de M. Le Verrier à M. Faye sur le théorie de Mercure et sur le movement du périhélie de cette planète." Comptes Rendus (Paris), 49, 379-383. Retrieved from https://archive.org/stream/comptesrendusheb49acad\#page/378/mode/2up

Levitt, L. S. (1978). Is the Photon a Double Helix? Lettere al Nuovo Cimento, 21, 222-223.

Lewis, P. J. (2007). Empty Waves in Bohmian Quantum Mechanics. British Journal for the Philosophy of Science, $58,787-803$.

LIGO. Retrieved from https://www.ligo.caltech.edu/

Lockwood, E. H. (1961). A Book of Curves. Cambridge University Press. Retrieved from http://www.aproged.pt/biblioteca/ABookofCurvesLockwood.pdf

Mancosu, P., \& Arana, A. (2010). Descartes and the Cylindrical Helix. Historia Mathematica, 37, 403-427. Retrieved from https://www.sciencedirect.com/science/article/pii/S0315086009000949

Maor, E., \& Jost, E. (2014). Beautiful Geometry. Princeton University Press, Princeton. ISBN-13:978-0-691-15099-4.

Nauenberg, M. (1993). Newton's Early Computational Method for Dynamics. Retrieved from https://core.ac.uk/download/pdf/82476251.pdf

Nauenberg, M. (2018). Newton's Graphical Method for Central Force Orbits. Am. J. Phys., 86, 765-771.

Nauenberg, M. (2018). Visiting Newton's Atelier before the Principia, 1679-1684. Arxiv: 1805.06871v.

Newton, I. (1687). The Principia. Mathematical Principles of Natural Philosophy. Translated by I. B. Cohen and A. Whitman. University California Press, Berkeley. ISBN 978-0-520-08816-0.

Newton, I. (1704). Optics: or, a treatise of the reflections, refractions, inflexions, and colors of light. Also two treatises of the species and magnitude of curvilinear figures. Palo Alto, Calif.: Octavo, ISBN 1-891788-04-3.

Parks, H. V., \& Faller, J. E. (2010). A Simple Pendulum Determination of the Gravitational Constant. Arxiv: $1008.3203 \mathrm{v} 3$.

Pedal curves properties. (2018). Retrieved from https://en.wikipedia.org/wiki/Pedal_curve

Pilot Wave Theory and Quantum Realism. (2016). Space Time, PBS Digital Studios. Retrieved from https://www.youtube.com/watch?v=RlXdsyctD50

Pilot Wave Theory. Retrieved from https://en.wikipedia.org/wiki/Pilot_wave_theory

Pioneer Anomaly. Retrieved from https://en.wikipedia.org/wiki/Pioneer_anomaly

Pladevall, X. O., \& Mompart, J. (2019). Applied Bohmian Mechanics: From Nanoscale Systems to Cosmology (2nd ed.). Jenny Stanford Publishing, ISBN 10-9814800104.

Pound, R. V., \& Rebka, G. A. (1959). Gravitational Red-Shift in Nuclear Resonance. Phys. Rev. Lett., 3, 439-441.

Pound, R. V., \& Snider, J. L. (1964). Effect of Gravity on Nuclear Resonance. Phys. Rev. Lett., 13, 539-540.

Quantum Gravity. Retrieved from https://en.wikipedia.org/wiki/Quantum_gravity

Regener, E. (1933). Der Energiestrom der Ultrastrahlung. Zeitschrift der Physik, 80, 666-669.

Roseveare, N. T. (1982). Mercury's Perihelion from Leverrier to Einstein. Oxford: University Press. ISBN 978-0-19-858174-1. 
Rothleitner, C., \& Schlamminger, S. (2017). Invited Review Article: Measurements of the Newtonian Constant of Gravitation, G. Rev. Sci. Instrum., 88, 111101-1.

Roveli, C. (2018). Physics Needs Philosophy. Philosophy Needs Physics. Found. Phys., 48, 481-491. Retrieved from https://arxiv.org/ftp/arxiv/papers/1805/1805.10602.pdf

Schumacher, A. (1999). Systematische Untersuchungen zur Messung der Newtonschen Gravitationskonstanten mit einem Pendelresonator. PhD Thesis, University of Wuppertal, WUB-DIS-99-13.

Schwarzschild, K. (1916). Über das Gravitationsfeld eines Massenpunktes nach der Einstein'schen Theorie. Sitzungsberichte der Königlich- Preussischen Akademie der Wissenschaften, Reimer, Berlin.

Seager, W. (2018). The Philosophical and Scientific Metaphysics of David Bohm. Entropy, 20, 493.

Smolin, L. (2017). Three Roads to Quantum Gravity. Basic Books, ISBN-10:0465094546.

Soldner, J. (1801). On the Deflection of a Light Ray from its Rectilinear Motion. Retrieved from https://en.wikisource.org/?curid=755966

Stávek, J. (2019). Super-Elastic Double-Helix Model of Photon. Huygens-de Broglie Particle on the Helical Path Guided by the Newton-Bohm Entangled Helical Evolute. Quantum of Magnetic Flux Based on the Mathematical Beauty of Newton, Lorentz, Einstein, Dirac, Gell-Mann, Schwinger, Polchinski and Witten (16.06.2019). Appl. Phys. Res., 11, 40-51.

Tests of General Relativity. Retrieved from https://en.wikipedia.org/wiki/Tests_of_general_relativity \#cite_note-Ein1916-3

Theo Murphy Meeting Issue. (2014). The Newtonian Constant of Gravitation, a Constant too difficult to measure? Philos. Trans A Math Phys. Eng.. Sci., 372(2026).

Thorne, K. S. (1995). Black Holes \& Time Warps. W.W. Norton \& Company. ISBN-10:0393312763.

Tombe, F. D. (2019). The 1855 Weber-Kohlrausch Experiment (The Speed of Light). The General Science Journal. Retrieved from https://www.scribd.com/document/294114501/The-1855-Weber-KohlrauschExperiment-The-Speed-of-Light

VIRGO. Retrieved from http://www.virgo-gw.eu/

Walleczek, J., Grossing, G., \& Pylkkanen, P. (2019). Emergent Quantum Mechanics: David Bohm Centennial Perspectives. Mdpi AG, ISBN-10:3038976165.

Watson, J. D. (2001). The Double Helix: A Personal Account of the Discovery of the Structure of DNA. Touchstone, ISBN-10: 9780743216302.

Weinstein, G. (2015). General Relativity Conflict and Rivalries: Einstein's Polemics with Physicists. Cambridge, Cambridge Scholars Publishing. ASIN: B01JXNM3MW.

Wien's Displacement Law. Retrieved from https://en.wikipedia.org/wiki/Wien\%27s_displacement_law

Wilczek, F. (2016). Physics in 100 years. Physics Today, 69, 32-39.

Will, C. M. (2006). The Confrontation between General Relativity and Experiment. Living Rev. Relativ., 9, 3. Retrieved from https://www.ncbi.nlm.nih.gov/pmc/articles/PMC5256066/

Williams, G. (2019). Unravelling the Double Helix. The lost Heroes of DNA. Weidenfeld \& Nicholson, ISBN-10: $147460935 \mathrm{X}$.

Wu et al. (2019). Progress in Precise Measurements of the Gravitational Constant. Annalen der Physik (Berlin), $531,1900013$.

Yates, R. C. (1974). Curves and their Properties. National Council of Teachers of Mathematics, ISBN: 10-087353039X.

Zwicky, F. (1929). On the Red Shift of Spectral Lines through Interstellar Space. Proceedings of the National Academy of Sciences of the United States of America, 15, 773-779.

\section{Copyrights}

Copyright for this article is retained by the author(s), with first publication rights granted to the journal.

This is an open-access article distributed under the terms and conditions of the Creative Commons Attribution license (http://creativecommons.org/licenses/by/4.0/). 